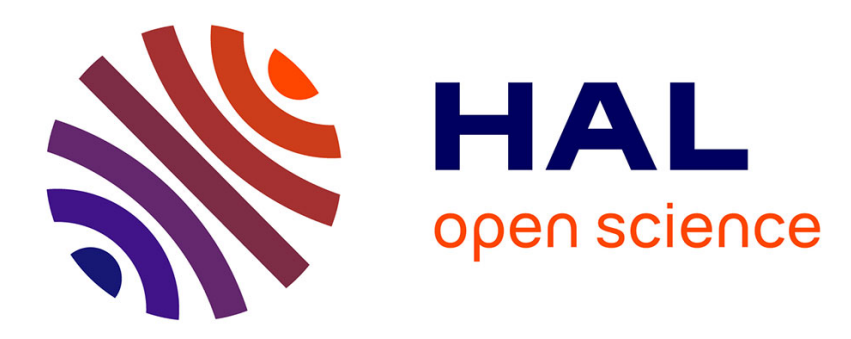

\title{
De la nymphe au complexe de la nymphette
}

Mareike Wolf-Fédida

\section{To cite this version:}

Mareike Wolf-Fédida. De la nymphe au complexe de la nymphette. Adolescence, 2008, 2 (64), pp.323337. 10.3917/ado.064.0323 . hal-01449592

\section{HAL Id: hal-01449592 \\ https://hal.science/hal-01449592}

Submitted on 30 Jan 2017

HAL is a multi-disciplinary open access archive for the deposit and dissemination of scientific research documents, whether they are published or not. The documents may come from teaching and research institutions in France or abroad, or from public or private research centers.
L'archive ouverte pluridisciplinaire HAL, est destinée au dépôt et à la diffusion de documents scientifiques de niveau recherche, publiés ou non, émanant des établissements d'enseignement et de recherche français ou étrangers, des laboratoires publics ou privés. 
La référence à la nymphette apporte un éclairage important sur la « jeunesse » du complexe d'OEdipe. Cette théorie a pris forme au fil de nosdiscussions, avant de s'installer comme une évidence dans la pensée de Pierre Fédida.J'essaierai de reconstituer nos débats avec le recul dequelques années. Il s'agissait de conversations de la vie courante, portantsur nos préoccupations intellectuelles respectives.

Tout d'abord, je voudrais remercier Maurice Corcos et PhilippeGutton de m'avoir sollicitée au sujet du commentaire de Pierre sur Lolita.Ce travail peut en effet rester énigmatique et il demande quelquesexplications. Pierre dit lui-même qu'il pensait présenter cette contribution dansun autre colloque. Mais, en fait, il reprend des idées qu'on lui connaît depuislongtemps. D'ailleurs M. Corcos fait lui-même le rapprochement au début avec « Le cannibale mélancolique » (Fédida, 1972) et L'absence (Fédida, 1978).Des textes anciens en rapport avec la perte et l'angoisse de la séparation.

\section{LE POINT DE DÉPART, L'IDÉE INITIALE}

Une lecture attentive aux mots (d'où l'insistance sur la traduction)de Lolita de Nabokov vient étayer l'idée, déjà présente chez Freud, du role particulier de la jeune fille.Il faudra faire attention à l'ambiguïté enfrançais :Fille=Tochter et Mädchen, c'est-à-dire la fille d'un parent(Tochter) ou la jeune fille en général (Mädchen). S'il y a une confusionpossible avec le sens, sa propre fille (Tochter), il vaudra mieux préciser lapetite fille ou la jeune fille. Chez Freud, il est question de la jeune fille dansla psychologie masculine (l'index dans les Gesammelte Werke, oeuvrescomplètes de Freud ${ }^{1}$, répertorie des références sur Mädchen, jeune fille,sur deux pages !), également chez J. Lacan (notamment dans «Lasignification du phallus » Lacan, 1958). Ce rôle, Pierre le reprend sous uneautre forme : la version animée dans « La tresse » (1999) et la versioninanimée dans «La relique et le travail du deuil » (1970). Mais hors cestextes antérieurs, l'élaboration se fixe sur les restes, les traces, lesempreintes comme témoignages silencieux de vie. L'idée nouvelle, c'estde considérer un état passager au lieu de la vie en entier tout en ayant lecaractère de trace. Il s'agit de faire apparaître un état passager (qui ne sefixe pas comme pour la pré-adolescence ou l'adolescence) se situant entrela féminité (ce qui n'est pas forcément la femme) et l'infantile (qui n'estpas nécessairement l'enfant). L'intrigue de Lolita arrive donc à point pournommer cette affaire : c'est une histoire de " nymphette ».

Une autre lecture a passionné Pierre tout autant :L'enfant éternel dePhilippe Forest ${ }^{2}$ Ces deux ouvrages, il les a littéralement épluchés. Ledernier a précédé la lecture de Lolita. Or dans le premier il s'agit d'un pèrequi voit sa fille mourir et qui la rend vivante à travers ce roman. Alors quedans le second, il s'agit d'un homme qui se rapproche d'une toute jeunefille en devenant son beau-père. La mort de la mère de la fille porte à soncomble son imagination, mais c'est aussi le début de grands

\footnotetext{
${ }^{1}$ Freud S., Gesammtregister. Gesammelte Werke chronologisch geordnet.

S. Fischer: Frankfurt am Main, 1968, pp. 342-344.

2 Forest Ph. (1997). L'enfant éternel. Paris : Gallimard.
} 
embarras.Elle devrait être à lui alors qu'il ne cesse de voir la fille s'éloigner. Dans

l'un «l'enfant qui meurt est éternel »", dans l'autre la fille a égalementdisparu, puisqu'elle est devenue « mère ».

Penser en termes antinomiques est, me semble-t-il, une

caractéristique chez Pierre (Wolf-Fédida, 2007). Il n'est donc passurprenant que l'histoire de la nymphette, venant comme uncomplement au complexe d'OEdipe, fasse allusion à la mort. Car il n'y a pas decomplexe d'OEdipe sans complexe de castration. Ce dernier génèrel'angoisse de la mort - la mort étant la séparation et la perte la plus brutale.

En 2001/2002, je venais de prendre mes fonctions en tant queprofesseur des universités et mon séminaire de recherche était sur le«vieillissement ${ }^{4}$. L'intitulé $d u$ dernier séminaire de Pierre était« Humain/Déshumain. L'oubli, l'effacement des traces, l'éradicationsubjective, la disparition $»^{5}$. Ces titres peuvent donner une impressionlugubre. Alors qu'il faut savoir que les intitulés de séminaires de recherché changent chaque année sur l'initiative du directeur de recherche et selonla logique de sa recherche. En ce qui me concerne, je pense que le terme «vieillissement » est une traduction malheureuse des termes anglais etallemand Aging et Altern. Ceux-ci indiquent un processus et donnent uneidée du devenir et du projet. Alors que cette connotation est complètementabsente dans la langue française. C'est la raison pour laquelle j'ai dûpréciser que le vieillissement est à entendre dans le sens « des âges de lavie ». Tout âge implique des transformations ou des crises telles qu'onl'entend pour l'adolescence. Pierre et moi, nous étions partis dans nosdiscussions sur la crise d'adolescence dans le vieillissement (les couplesqui se séparent à la retraite ou divorcent à quatre-vingts ans ; la crisecomme moyen autothérapeutique ; comme revitalisation, etc.). Mais nousn'avions pas la même idée de la structure de la chose ce qui a animé delongues discussions entre nous.

M'intéressant donc à l'altération de la vie psychique dans desaffections neurologiques, immunologiques et organiques en les étudiantdans le sens du « vieillissement »- terme si inadéquat que l'annéesuivante j'ai parlé de «maturation » en l'opposant à la «régression », jecernais davantage les processus psychiques. Car il est un fait que l'hommene se voit pas vieillir tout comme l'enfant ne se voit pas « petit ». Quantà Pierre, il avait repris la dépression (Fédida, 2001a) et " l'informe »(Fédida, 2000) et il s'est intéressé à la psychopathologie plutôt sous formede « restes »du psychique ou de « survivance ». Il a considéré davantagele psychique comme phénomène, renouant avec sesreferences phénoménologiques. Le féminin et le masculin en sont également lestraits (Fédida, 1973), à considérer à la fois comme " reste ", dans le sensde ce qui résiste au complexe d'OEdipe, et comme une force animant lesrapports humains. Pierre a notamment intitulé son dernier livre (inédit)Entre hommes et femmes.

Il me taquinait parce que je travaillais sur le «vieillissement », alorsqu'on a plutôt tendance à le fuir, et nous nous sommes obligés à définir ceque nous entendions au juste par ce terme. Je venais de faire paraître unouvrage auquel S. Lebovici avait collaboré avec son dernier texte dont le titreétait justement «Le vieillissement »

\footnotetext{
3 Ibid., p. 383.

${ }^{4}$ Séminaire : Psychanalyse et vieillissement, «Troubles neurologiques, génétiques et conflits psychiques en comparaison avec des modèles de la psychose ».

5 Publié dans Fédida, André et al., 2007.
} 
(Eustache, Wolf, 2002). Ce texte estassez dur et il nous a fait réfléchir. La question que nous nous posions portaitsur ce qui irait à l'encontre du vieillissement. Puisque celui-ci est inéluctable,il est plus intéressant de s'interroger sur la lutte que l'on mène pour l'écarter.

Nous pensions que le vieillissement se traduit par des forcesdépressives. Celles-ci seraient mises en suspens par l'animationpsychique (sous forme modérée, par exemple, l'intrigue dans la famille ousur le lieu du travail ou alors, sous forme excessive, dans l'expressionpsychopathologique, épuisante pour les autres mais qui conserve sonauteur). Cette animation du psychique est garantie par le complexed'OEdipe. J'avais notamment observé dans ma recherche que les maladesd'Alzheimer présentent des réactions très lisibles au niveau du complexe d'OEdipe comme chez les enfants. D'où notre discussion pour savoir si lecomplexe d'OEdipe auquel incombe le rôle de structurer la vie psychiqueest altérable par le vieillissement. Et s'il y avait une altération dans quell sens fallait-il la définir ? Car les relations humaines peuvent ne pasévoluer ou également se gâter. Bien sûr, nous pensions au cas où unepatiente âgée de soixante-dix ans se plaignait toujours de sa mère bientôtcentenaire comme si elle ne voulait pas se rendre à l'évidence que celleci était réduite au fauteuil roulant et qu'elle pouvait suivre le même sortencore plus tôt que sa mère. Le transfert resterait-il toujours le meme inaltérablement ? Les plaintes et les revendications ne mûrissent-ellesjamais ? Et puis, qu'en est-il de la sagesse soidisant acquise avec l'âge?

Il faut convenir qu'il est curieux d'envisager qu'il n'y aurait aucunematuration ou altération de la structure psychique malgré le corpsvieillissant. À moins que ce ne soit pour démentir ce vieillissement que lamême structure est entretenue au point que ce qui semble avoir étéinchangeable est déjà affecté par la régression. Pour reprendre l'exemplede Lolita : au moment où Humbert devrait changer, et alors qu'il chercheune chambre en attendant un nouveau poste, il croise le regard de la jeunefille et régresse à la perception infantile de son premier amour. S'il épousela mère de la jeune fille, c'est pour satisfaire extérieurement la situation.Mais son état affectif ne présente aucune maturité pour ce mariage. Lafemme mature le dégoûte.

Les travaux sur l'enfance ou l'adolescence montrent bien quel'individu se positionne différemment dans le complexe d'OEdipe selonl'âge de sa vie. Ce n'est pas le complexe d'OEdipe en soi qui change, maisquelque chose de l'ordre de l'investissement. Par exemple, le petit Hansqui veut épouser sa mère et prévoir une petite pièce pour son père dans sagrande maison n'aura plus les mêmes objectifs plus tard - ou alors ceserait inquiétant. En tant qu'adolescent " normal ", il « enverraitpromener $\gg$ sa mère et son père tout en restant très susceptible à l'égard dela moindre inattention. Pourquoi ne pas considérer un changement pour lapersonne vieillissante ? Et tant qu'on y est, pourquoi ne pas examiner cechangement selon le versant féminin ou masculin selon les âges de la vie ?Puisque le garçon et la fille ne se comportent pas de la même manière dansle complexe d'OEdipe, alors on peut imaginer que l'homme et la femmese voyant vieillir et en étant donc confrontés au complexe de castration àleur insu, trouveraient leurs propres façons à elle ou à lui pour rééquilibrerla vie psychique.

Pierre s'est intéressé au phénomène de la reviviscence de la viepsychique dans le complexe d'OEdipe. En quelque sorte, l'aspect durajeunissement au lieu du vieillissement. Dans cette optique, il devientplus clair que le complexe d'OEdipe sert à tenir l'affectivité éveillée. C'està ce propos que Pierre avance donc le profil de la nymphe. En laissantlibre cours aux associations, il passe en revue certains cas cliniques. Bienévidemment, j'ai voulu tenter de comprendre cette histoire de 
nymphe,redoutant qu'elle ne soit accessible qu'à la perception masculine. Maisavec un peu « d'entraînement », je parvenais également à distinguer dansnotre entourage des individus pouvant être qualifiés de «nymphettes ».Toutefois, il faudra faire attention, car dans le passage de «nymphe » à« nymphette » se traduit, pour ainsi dire, la perspective diagnostique. Jel'expliquerai plus loin.

La «nymphette » devenait, grâce au texte de Lolita, quasiment « empirique ». Dans l'optique d'une catégorie nosographique, il devenaitpossible d'élaborer une théorie (la nosologie) : comment devient-onnymphette ? Est-ce un symptôme et à quoi cela sert-il ? Existe-t-il unremède et est-il utile d'y remédier ?

Ce développement est intéressant parce qu'il contribue à ladistinction des sexes selon leur fonctionnement psychique en reprenant « Quelques conséquences psychiques de la différence anatomique entreles sexes » de Freud (1925). Mais aussi, cette réflexion montre que letransfert et le contre-transfert ne travaillent pas l'inconscient de la meme manière selon les sexes.

Le complexe d'OEdipe génère le transfert. Or si on réfléchit entermes de complexe de la nymphette (je le construirai plus loin), il est bienévident que l'équivalent n'existe pas de cette manière chez la femme.L'analogie est difficile. L'idéalisation du masculin dans le garçon prépubèren'est pas aussi évocatrice pour la femme qui mûrit que l'image dela jeune fille chez l'homme d'un certain âge. En revanche, l'hommepédophile qui s'intéresse au garçon, choisira ce dernier de preference avant la puberté. Les pédophiles ont souvent horreur des premièresapparitions de la maturation sexuelle. Alors que cela ne gêne nullementpour Lolita, bien au contraire. Elle est à « l'aube » de la maturité sexuelle- ce qui la rend intéressante. L'analogie est donc difficile, il s'agit dansd'autres cas de figure d'un mouvement psychique différent ou detemporalités différentes. Dans l'histoire de la nymphette se dégage à la foisla régression, l'idéalisation et un sentiment de puissance, de vitalité. Il peutparaître surprenant qu'une petite créature puisse mettre en oeuvre tout cela.

Sur la cheminée de la chambre trône le livre de Claude Monet,Nymphéas ${ }^{6}$.Pierre l'a installé en décorant la chambre il y a longtemps,peut-être en 1988, date de la parution de l'ouvrage. Monet, en vieillissant,s'était retiré du monde parisien pour peindre. En le comparant auxpeintres proches contemporains, on remarque : «Ces travaux pourraientmême inclure des compositions telles que celles de Renoir ou de Cézanne,des scènes de baigneuses dans des paysages d'Arcadie imaginaires (lemot " nymphéa " vient de " nymphe "). Le fait que Monet ait souhaité selancer dans la peinture de personnages, ce qu'il n'a pas fait, suggère unesignification allégorique des Nymphéas ${ }^{7}$.

Pourquoi alors consacrer tout son art à reproduire des nénuphars ?

On trouve le témoignage suivant : "Vers 1889, il avait decide d'abandonner pour ainsi dire le paysage ; il en avait parlé à Renoir et à sesautres amis qui lui avaient fortement conseillé l'étude de la figure : il étaitvenu à Paris chercher un modèle, il trouva et engagea une fille très bien, quiavait consenti à venir demeurer à Giverny ; mais quand il retourna chez lui,sa femme lui dit : "Si un modèle entre ici, je sors de la maison ". C'estpourquoi nous n'avons pas connu Monet, peintre de portraits et de figures $»^{8}$.

\footnotetext{
${ }^{6}$ Monet Cl., Nymphéas. New-York : Hugh Lauter Ass. Inc., 1988.

${ }^{7}$ Ibid., p. 12.

8 Ibidem.
} 
En revanche, on y raconte également que Monet a aperçu l'oeuvrede Morisot, une copie d'une peinture de Boucher, montrant « deuxnymphes couchées dans une mer de nuages lumineux. C'était un étonnantprécédent des Nymphéas $»^{9}$.

En suivant les définitions, la nymphe est une « déesse au ranginférieur » au même titre que la naïade ou l'océanide. Il existe égalementun sens anatomique, " les petites lèvres de la vulve ». En revanche, lanymphette est « une très jeune fille au physique attrayant. Adolescente auxmanières aguicheuses, à l'air faussement candide ». Le passage du divinau vulgaire est intéressant dans ce faux diminutif, par le suffixe « ette ».

Quand on pense à la désignation d'une femme mûre et aguicheuse comme« vamp », " type de femme fatale et irrésistible », celle-ci se décline en unverbe « vamper », « séduire par des allures de vamp ». Alors que ledictionnaire ne prévoit pas le verbe «nympher ». Le nymphée est la « grotte naturelle ou artificielle où jaillissait une source, une fontaine,sanctuaire consacré aux nymphes $»^{10}$. Décidément le rapprochement entrele sacré et le futile se condense chez la jeune fille.

Pourtant nous nous sommes servie de l'expression « nympher » enallusion à « vamper ». Il aurait fallu dire «nymphetter »! Il y a deux choses quisont à retenir : la condensation des contraires (sacré et vulgaire) et le jeune âge.Quand Pierre parle de la nymphe qui devient nymphette, il s'agitd'un renversement dans la temporalité. Car selon le dictionnaire, lanymphette est plus jeune que la nymphe. Puis, c'est également unbouleversement des valeurs : l'innocence sacrée bascule dans le mondain.

Alors que dans l'utilisation que Pierre fait de la «nymphette », c'est unétat arrêté qui sort d'un coup chez une jeune fille et qui peut être passagerou perceptible pendant toute sa vie. Le fonctionnement de nymphette peutaussi exister chez une fille de dix ans comme chez une jeune femme devingt-cinq ans, de cinquante-cinq ans et peut-être davantage. La modeaméricaine de travestir des jeunes filles qui sont presque des bébés commemannequins montre l'élasticité de la représentation de l'âge de laséduction dans l'autre sens. Un rapprochement avec la nymphomanien'est pas opportun. Car celle-ci est caractérisée par des femmesvolontaires et entreprenantes dans les conquêtes sexuelles. Cela n'est pasle cas chez la nymphette qui joue son immaturité tout en provoquant demanière passive. Elle ne sait pas trop quoi faire de ses conquêtes. En toutcas, il ne faut pas chercher de responsabilité chez elle. Elle serait plutôtdans une relation de dépendance ou de mécénat.

Les jeunes filles peintes par Balthus sont particulièrementévocatrices de la condensation des sens contraires commemûre/immature, sacré/vulgaire, enfant/adulte. Elles sont présentéescomme des jeunes filles au point d'éclosion de la nymphette. Dans untexte intitulé «Autour de Balthus »", Pierre écrit ceci : «Plans et angles.L'angulaire est un angle de vue dans le cadre d'une fenêtre. Il accroît alorsle rapport du corps (au paysage : le corps nu ou à moitié dénudé des jeunesfilles apparaît irréalisé, dans un temps suspendu - ce qui intensifie lacruauté de la vue. Décalque du corps sur le paysage ou inversement.Corps en décalque parce que ce seraient les tonalités et modalités dupaysage (est-ce un paysage ou est-ce un décor ?) qui détiendraient lesmodalités de l'érotique. Ouverture du rideau de la fenêtre : opéra quiinstaure le corps féminin comme un corps objectivé dans un regardinvisible. Certes tensions physiques à l'abri de l'expressivité (comme lesreprésentations dans le rêve). Rilke et Jouve : l'âme (ou la quintessence) émaned'un corps fait seulement de

\footnotetext{
${ }^{9}$ Ibidem.

${ }^{10}$ Les définitions émanent du Petit Robert.

11 Manuscrit. J'ignore s'il a été publié.
} 
la lumière. Physique de l'âme ou de la langue. "

La mise en scène des jeunes filles est alors importante. La peinture qui se joue comme un théâtre.

Il poursuit : «Mais on peut aussi voir en quoi Balthus, par son procédéde décalque de certaines oeuvres du quattrocento (notamment), tente derévéler l'alliance entre nudité et paysage, alliance entre angulaire et lumière.Événement : ce qui a eu lieu ou n'a pas encore lieu reste dansl'ombre. Quelque chose ne se montre pas et est terrifiant. Événement nonnommable. Auteur de l'événement et spectateur ne font qu'un. Lapeinture de Balthus rend cet événement onirique ou intemporel. Ilcontinue à avoir lieu.Les jeunes filles de Balthus ne sont que des enfants et ellesressemblent à des femmes.Corps violemment mis à nu : projection à l'intérieur de la pièce.Renversement. Apparence de position d'offrande mais anesthésié.La mort est toujours présente sous l'apparence d'une couleur.Peut-on dégager la cruauté de la lumière balthusienne de cette cruautéangulaire de la mort ?»

Voici donc la condensation des contraires : enfant/femme,offrande/anesthésie, nudité/mort... L'image est tellement soignée qu'elleen devient inquiétante :

«Ce qui est terrifiant c'est la désincarnation de la femme par un actedu peintre (impuissance). Le terrifiant maintient en suspense l'acte sexuelsans le manifester comme tel. Et l'inquiétante étrangeté de ce terrifiantréside dans une apparence subvertie et qui ne dit pas son nom. RegarderBalthus comme on écoute un rêve.Comment la pureté de l'apparence (corps, paysage, lumière) peutelleêtre ici si proche de la perversion ? La fonction du leurre ou del'illusion. Prêter attention à l'immobilité tendue. Les corps restent dansl'orbite de la vue : ils n'en sortent pas.C'est peut-être l'exact contraire de la spiritualité de Fra Angelico(l'incarnation) qui accorde la lumière et l'espérance au verbe et exige le mystère.»

Cette inquiétante étrangeté chez la jeune fille à l'éveil du féminin vajusqu'à évoquer la mort qu'il s'agit de maîtriser à travers sa mise en scène.Pour mieux comprendre cela, il faudra revenir en arrière aux travaux deFreud et de J. Lacan sur la figure de la jeune fille.Quand on consulte l'index allemand des oeuvres complètes de Freud $^{12}$ il y a de quoi être surpris. Le registre des oeuvres complètes deFreud a été indexé et tous les mots que Freud a employés dans ses textesont été répertoriés en allemand. Pour les lecteurs allemands, le vocabulairede la psychanalyse comporte tous les mots que Freud a utilisés. Il s'avèreque les références sur la jeune fille (Mädchen) couvrent deux pages, alorsque le garçon (Junge est inexistant !), Knabe, n'occupe qu'une page, lesréférences concernant aussi souvent la jeune fille. On peut se demander ce qu'elle a de plus pour être si souvent citée ?

Mais elle est citée pour ce qu'elle a de moins ! Ce serait un autre travailque de commenter toutes les références de Freud à la jeune fille. Enrésumé, il s'agit de références relatives au complexe de castration et celaà toutes les étapes (phase prégénitale, phase phallique, puberté) etnotamment dans le fantasme d'être battu. J'y reviendrai plus bas, avec unautre texte de Pierre, contemporain de Lolita et de " Autour de Balthus »et qui l'éclaire bien.

Dans son texte «La signification du phallus », J. Lacan prendégalement le soin d'expliciter en quatre points en précisant que les faitscliniques « démontrent une relation du sujet au phallus qui s'établit sans égardà la différence anatomique des sexes et qui est de ce fait d'une interpretation spécialement épineuse chez la femme et par rapport à la femme $»^{13}$.Plus tard, il reprendra l'expression de Freud d'une « autre

\footnotetext{
12 Freud S., Gesammtregister. Op. cit.

13 Lacan, 1958, p. 686.
} 
scène »(ein andere Schauplatz) «que Freud à propos des rêves désigne commeétant celle de l'inconscient $»^{14}$. J. Lacan développera le sujet du désir dansle langage. Ce passage du texte de J. Lacan fait penser à la description dePierre dans « Autour de Balthus » qui illustre par la mise en scène enpeinture cet écart du signifiant au signifié dans lequel se situe le désir.

Dans un autre texte, "L'angle vif », un hommage à W. Granoff,d'abord prononcé sous forme de conférence à l'APF, puis publié, Pierrereprend l'ouvrage de celui-ci, La pensée et le féminin (Granoff, 1976) eny développant « la pensée du féminin ». Après un commentaire sur labisexualité psychique, Pierre dit ceci : «Dans " On bat un enfant", lefantasme dans sa nature hystérique fait coïncider en un même mouvementle passif et l'actif, sans que l'on puisse pour autant attribuer à l'un leféminin et à l'autre le masculin. L'intérêt de conjoindre fantasme etinterprétation est précisément celui de rendre possibles dans le langage lesopérations de transformation entre passif/actif, féminin/masculin etd'éviter de la sorte les objectivations catégorielles » (Fédida, 2001b).

Je pense que ce passage est très important. Ce fantasme est rattachéchez Freud à l'indexation de la jeune fille. Autrement dit, le complexe decastration est surtout celui de la fille !Puis, le garçon, l'homme ou lemasculin s'y rattachent. (En quelque sorte, ils y reviennent commel'abuseur se délecte de sa victime tout en l'idéalisant.)Manifestement, la jeune fille est idéalisée dans la psychanalyseparce qu'elle a un rôle particulier à jouer dans la vie psychique de l'êtrehumain. Elle donne la clé de compréhension de «l'énigme de laféminité » explique Pierre. Dans un autre texte «Quelque chose durapport de la psychanalyse au féminin ${ }^{15}$, Pierre va jusqu'à expliquer, enréférence à W. Granoff, que la femme est toujours restée une énigme pourFreud et que c'est ainsi qu'elle exerce son emprise.À y réfléchir, cela revient à dire que tout ce que l'on soutient sur lajeune fille reflète une certaine vérité, mais c'est encore plus parlant pourcelui qui l'observe. La nymphette n'intéresse que l'homme disposé às'enivrer de ses charmes. La nymphette ne « nympherait » (néologismeindispensable) pas dans le vide si elle n'était pas intéressée par l'intrigue et son résultat. Comment le destinataire peut-il se défaire de l'emprise duféminin ? La nymphette est à la fois la solution pour se défaire de cetteemprise et un leurre. C'est une situation paradoxale.

Examinons un autre texte, dans « La sexualité femme ${ }^{16}($ au lieu dedire sexualité féminine), toujours en référence aux publications de W. Granoff,notamment «Quitter Freud » ${ }^{17}$, l’omniprésence de la femme est dénoncée.

" Il ne s'agit pas d'accroître une thématique en survalorisant lasexualité féminine au service des parti-pris. Il s'agit plutôt d'amplifierl'image d'une terreur qu'inspire aux hommes la sexualité de la femme.Cette amplification de l'image n'est pas tant l'amplification de l'image dela femme : c'est - comme lorsqu'il s'agit de la “ méduse "- la puissancevisuelle qu'acquiert la sexualité-femme au point de sidérer la pensée ${ }^{18}$.

\footnotetext{
14 Ibid., p. 689.

15 Un manuscrit, probablement non publié, car les notes n'ont pas été complétées. Vraisemblablement il s'agit d'un texte intermédiaire ayant servi à la fois la conférence et la publication.

16 Probablement pas publié et servant comme texte intermédiaire à la publication suivante.

17 Paru dans L'inactuel, 1996.

18 Manuscrit vraisemblablement inédit.
} 
Nous allons retrouver le fil rouge avec un autre texte, cette fois-cipublié, « Le passé d'un vu, la castration instauratrice » (Fédida, 2001c)dont l'intitulé nous remet au coeur de la problématique du clivage. Enpartant du texte de Freud (1938) «Le clivage du moi dans le processus dedéfense », Pierre précise pour la fille : « Quant au pénis et à l'énoncé deson manque, la fille - nous dit Freud - est d'emblée plus exigeante avecles mots : elle l'a vu, elle sait qu'elle ne l'a pas, elle le veut. Rien desemblable chez le garçon qui, par un effet d'une arrangeante irrésolution,pactise avec le temps et avec un esprit de phrase qui ménage l'allusion- façon de retarder la décision. Car c'est une sorte de courage qu'il est fait appelpour nommer ce qui est. La castration est toute entière dans cette decision de donner lieu optique et de pensée à la fente du clivage dans le moi. »

Si l'on considère que la fille est plus courageuse, on est amené àpenser que l'éveil de sa féminité va la mettre de nouveau et de manièreaccrue devant l'évidence de ce qu'elle n'a pas. Si elle arrive à en jouer,c'est comme si elle transformait la béance en signification phallique. Lepouvoir de la séduction qu'elle en tire est mis en analogie avec la théoriesur le phallus qui poussera plus tard.

P. Mendousse, dont les travaux sur l'adolescence remontent à 1910,définit l'âme de l'adolescente en précisant les différentes étapes etchangements d'attitude depuis l'entrée dans l'adolescence jusqu'à lamajorité (vingt et un ans). Cet ouvrage est une curiosité à mon avis. Je l'aidernièrement parcouru à la recherche d'une observation allant dans le sensde la nymphette et de la nymphe. P. Mendousse qualifie la puberté comme« L'âge d'indécision », "Les vaines tendresses ", "L'esprit romanesque »et «La plasticité mentale »; et il décrit les particularités des situationsaffectives selon l'environnement institutionnel, idéologique et intellectuel.Il signale également le caractère emprunté et la fluctuation des sentimentsqui sont typiques de cette étape. En cas de persistance, ils constitueront labase de la plupart des maladies mentales à l'âge adulte.

«En attendant, les unes et les autres ne sont encore que des enfantset, comme les enfants, elles ont besoin d'être aimées plutôt que d'aimer.C'est en vain que l'instinct d'imitation, joint au désir de paraitre degrandes filles, les pousse à essayer toutes les formes du sentiment : si elless'émeuvent si facilement, c'est que dans leur personnalité, fonctions ettendances sont dans un devenir incessant ; de l'enfant qui s'en va à lafemme qui s'approche, un va-et-vient confus s'établit, avec des arrêts etdes accélérations brusques dont elles ne peuvent prendre conscience que sous la forme d'émotions $» 19$.

P. Mendousse ne parle pas de nymphette, mais en comparant cesdescriptions avec Lolita, il semblerait que la nymphette résiste à cesfluctuations pour les érotiser trop rapidement. Ou alors elle croise un regardqui établit un «arrêt sur image » au cours d'une de ces fluctuations. Théâtralepour les uns, elle se placerait comme " l'objet du désir » pour les autres.La beauté du diable est un moment culminant après l'apparition dela première menstruation dans l'épanouissement de la jeune femmedécrite par M. Francillon et repris par P. Mendousse ${ }^{20}$. Il s'agit d'unmoment précis dans la vie de la femme où il s'agit de se maintenir le pluslongtemps possible et de descendre le plus lentement possible.

La comparaison s'impose avec un autre texte contemporain de cestravaux, celui de Freud, «Le clivage du moi dans le processus dedéfense ». Il montre les différents registres de l'observation. L'histoire dela nymphette s'inscrit dans une

\footnotetext{
${ }^{19}$ Mendousse, 1910, p. 115.

20 Ibid., p. 275.
} 
observation de l'inconscient vu comme «l'autre scène » déjà évoquée plus haut et elle n'est compréhensible qu'àtravers le complexe de castration.Je dirais qu'on peut parler d'un complexe de nymphette qui serattache fondamentalement au complexe de castration.L'utilité de cettedénomination dans la théorie psychanalytique consiste à démontrer que ledésir repose sur le partage du complexe de castration.

Je pense que nous n'avons pas encore suffisamment insisté sur lesdifférentes réécritures du complexe d'OEdipe. Le complexe de lanymphette est aussi une version masculine par rapport à la crise(chômage, milieu de la vie, maladie, vieillissement, etc.). Nous tenons unmodèle de réécriture avec les propositions de P.-C. Racamier pour lecomplexe d'OEdipe dans la psychose. Il a expliqué que la psychose leréinvente en donnant la version de l'abolition de la filiation ou en renversantles rôles des figures parentales (confusion entre la mère et le père).

L'histoire de la nymphette nous invite à concevoir le complexe d'OEdipede manière plus ouverte et à le préciser davantage. Elle ouvre à la discussion.Elle n'a pas pu être approfondie à cause de la disparition soudaine de Pierre,mais l'idée a été suffisamment élaborée pour être poursuivie sur sa lancée.Si on replace cette discussion dans un cadre plus large, on peut sedemander à quoi sert le complexe d'OEdipe. D'un point de vue clinique, ilanime la vie psychique. D'un point de vue théorique, c'est un paradigmepour éclairer la métapsychologie et en particulier sous l'aspect du transfertet de la structure psychique. La nymphette vue depuis le complexed'OEdipe est une " version adolescente », qui peut faire irruption à toutâge. De la fille bébé-mannequin jusqu'au point de maturité où la jeunefille attend son chevalier servant, ou le complexe de la nymphette secristallise ou se « gèle » (expression de Pierre). Mais il peut aussialimenter tout simplement le jeu fantasmatique dans la vie quotidienne.

Cette version bouscule nécessairement les représentations du temps dansle complexe d'OEdipe. Peu importe si nous parlons en termes devieillissement ou de rajeunissement.Il n'existe pas de nymphette sans un destinataire. L'un et l'autretranscendent les représentations du temps et le rôle qui incombe à l'êtrehumain selon l'âge de sa vie. Les sentiments évoluent en tournant le dosaux exigences de la société - une révolte qui caractérise l'adolescence.

\section{BIBLIOGRAPHIE}

EUSTACHE F., WOLF M. Éds. (2002). Trouble neurologique, conflit psychique.Paris : PUF. FÉDIDA P. (1970). La relique et le travail du deuil. Nouvelle Revue de Psychanalyse, 2 : 249-254.

FÉDIDA P. (1972). Le cannibale mélancolique.Nouvelle Revue de Psychanalyse, 6 : 123-127. FÉDIDA P. (1973). D’une essentielle dissymétrie dans la psychanalyse.Nouvelle Revue de Psychanalyse, 7 : 159-166.

FÉDIDA P. (1978). L’absence.Paris : Gallimard.

FÉDIDA P. (1999). La tresse. In : M. Schaeffer, M. Cournut-Janin, S. Faure-Pragier,

F. Guignard (Éds.), Clés pour le féminin. Femme, mère, amante et fille. Monographie de la Revue Française de Psychanalyse. Paris : PUF, pp. 135-141.

FÉDIDA P. (2000). L'informe agissant, la mise en oeuvre de l'informe.In :L 'art médecine. Actes du colloque.Paris : Réunion des Musées Nationaux.

FÉDIDA P. (2001a). Les bienfaits de la dépression.Paris : Odile Jacob.

FÉDIDA P. (2001b). L’angle vif.In :L'inactuel. En lisant Wladimir Granoff.Paris : Circé. FÉDIDA P. (2001c). Le passé d'un vu. La castration instauratrice. Libres Cahiers pour la Psychanalyse, 4.

FÉDIDA P., ANDRÉ J. et al. (2007). Humain/déshumain. Pierre Fédida, la parole de l'oeuvre. Paris : PUF, Petite bibliothèque de Psychanalyse. FREUD S. (1925). Quelques conséquences psychiques de la différence anatomique entre les sexes. In :la vie sexuelle. Paris : PUF, pp. 123-132.

FREUD S. (1938).Le clivage du moi dans le processus de défense.In :Résultats, idées, 
problèmes, II. Paris : PUF, 1998, pp. 283-286.

FREUD S. Gesammtregister.Gesammelte Werke chronologisch geordnet. Frankfurt am Main : S. Fischer, 1968.

GRANOFF W. (1976). La pensée et le féminin. Paris : Les Éditions de Minuit.

LACAN J. (1958). La signification du phallus. Die Bedeutung des Phallus. In :Les écrits. Paris : Seuil, 1966, pp. 685-695.

MENDOUSSE P. (1910). L'âme de l'adolescente.Paris : Félix Alcan, 1936.

WOLF-FÉDIDA M. (2007). Faire et défaire. Penser en termes antinomiques.In : P. Fédida,

J. André et al., Humain/déshumain. Pierre Fédida, la parole de l'oeuvre. Paris : PUF, Petite bibliothèque de Psychanalyse. 